\title{
A robust grid current controller with grid harmonic and filter resonance damping capabilities using a closed-loop admittance shaping
}

\author{
Jorge Pérez, Santiago Cóbreces \\ Department of Electronics \\ Universidad de Alcalá \\ Email: jorge.perez@depeca.uah.es Email: xwa@et.aau.dk, fbl@et.aau.dk \\ Xiongfei Wang, Frede Blaabjerg \\ Department of Energy Technology \\ Aalborg Universitet
}

\author{
Robert Griñó \\ Inst. of Industrial and Control Engineering.(IOC) \\ Universitat Politècnica de Catalunya (UPC) \\ Email: roberto.grino@upc.edu
}

\begin{abstract}
This paper presents a grid current control of a grid connected Voltage Source Converter (VSC) with an LCL filter. The control method enables to shape the input admittance of the converter in addition to track a given current reference. By specifying a low resistive admittance profile, a suitable controller is obtained by using an $\mathcal{H}_{\infty}$ synthesis, which will actively damp the filter resonance and the harmonics/inter-harmonics from the grid in a wide frequency range. Additionally, the proposed system presents good robustness with respect to grid uncertainty.
\end{abstract}

\section{INTRODUCTION}

This work presents a new grid current controller structure for a Voltage Source Converter (VSC) connected to the grid through an LCL filter. The topology is very common in renewable energy source based power plants, as it has superior output performance. However, the resonant behaviour of the LCL filter presents some drawbacks for its effective and stable current control from the points of view of power quality and system robustness:

From the former point of view, system resonances -in grid or filter- may lead to system oscillations, and even to instabilities of the controlled grid current [1], [2]. Many papers have focused on design of the resonance dampers that improve the overall current dynamics. They can be classified into active [2]-[9] and passive [10]-[14] damping techniques. A related issue is the effect of grid voltage harmonics and inter-harmonics in the control loop. The most usual approach is to design the current controllers with harmonic rejection capabilities, mainly by using PR (proportional + resonant) controllers tuned at the most common harmonics in order to reject them [15]-[17]. However, the harmonic rejection effectiveness depends on different factors, such as the model uncertainties, the discretization process and the presence of computational delays in the system, which may result in ineffective harmonic damping [18]. Additionally, the use of high feedback-gain controllers may lead to poor stability margins [19], and its stable operation becomes more difficult as the harmonics to be rejected approach to the filter resonance [20].

From the robustness point of view, it is known that the stability of the control loop of the LCL-filtered grid-connected
VSC may be compromised by the uncertainty in the grid equivalent impedance, which may lead to the uncontrolled displacement of resonances inside the control band. Several approaches to obtain robust controllers have been explored in the related literature [21], [22] being achieved at the cost of a conservative performance -control bandwidth- reduction, as a consequence of the need complying with Small Gain Theorem [23].

This paper translates the aforementioned problems into the frequency domain input admittance specifications, following the design procedure presented in [24]. This recent method allows to shape the input admittance transfer function of a VSC, in both modulus and phase, while maintaining tracking performance at selected harmonic frequencies.

This new capabilities open the door to face the robustness problem from an alternative point of view: in stead of the classical approach, based on the fulfilment of the Small Gain Theorem, it allows to face the problem trying to comply with certain input admittance conditions described in Middlebrook's criterion [12], [25], which may decrease the closed-loop performance reduction induced by uncertainty. If that admittance, additionally, is low out of the fundamental frequency, the converter will present good inter-harmonic behaviour. That condition also implies a good damping of the LCL-filter resonance [26]. The proposed controller will, in addition, track a given current reference in a band around the fundamental frequency.

The rest of the paper structure is as follows: Section II serves as a theoretical background to the rest of the paper, modelling the considered system and analysing its stability in both stand-alone and grid-connected conditions. Section III explains the proposed method and its limitations, in addition to give some guidelines for the design parameters tuning. Section IV presents the achieved experimental results in both time and frequency domain. It demonstrates the damping capabilities of the proposed method of both LCL filter resonance and grid voltage -inter-harmonics effect. Finally, it analyses its robustness for different grid impedances. The paper concludes with Section V. 


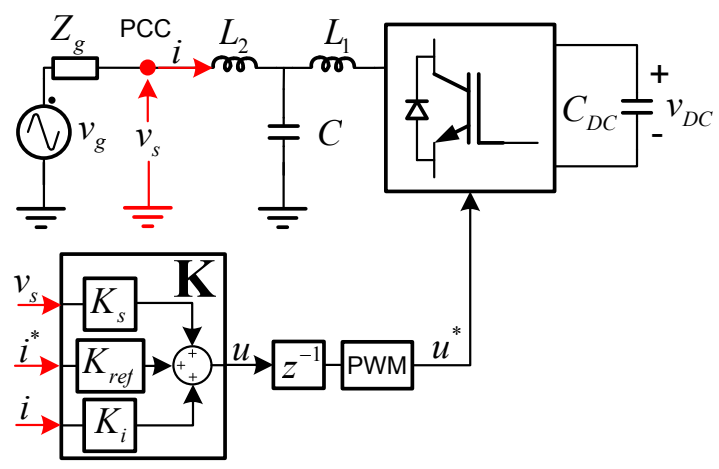

Fig. 1. Simplified single phase equivalent of the considered system.

\section{THEORETICAL BACKGROUND AND OBJECTIVES STATEMENT}

\section{A. System modelling}

Fig. 1 shows a single-phase equivalent of the considered system: grid current $i$ control of a grid-connected VSC with an LCL filter. This single-phase system is considered for both modelling and controller design procedure. Obtained controller can be easily translated and implemented in a three-phase system using $\alpha \beta$ stationary reference frame transformations [27] for its input and output signals.

Open-loop grid current can be expressed in Laplace domain as follows:

$$
I(s)=G(s) U(s)+G_{d}(s) V_{s}(s),
$$

where $I(s), U(s)$ and $V_{s}(s)$ are the grid current, controller output voltage and point of common coupling (PCC) voltage, respectively, and $G(s)$ and $G_{d}(s)$ are the open-loop commandto-output and admittance transfer functions;

$$
\begin{aligned}
& G(s)=-\frac{1}{s C\left(R_{1}+s L_{1}\right)\left(R_{2}+s L 2\right)+R_{f}+s L_{f}} \\
& G_{d}(s)=\frac{s C\left(R_{1}+s L_{1}\right)+1}{s C\left(R_{1}+s L_{1}\right)\left(R_{2}+s L 2\right)+R_{f}+s L_{f}}
\end{aligned}
$$

where $C, L_{1}$ and $L_{2}$ are the filter capacitor and the converter-side and grid-side filter inductance values, respectively, and $R_{1}$ and $R_{2}$ are their respective resistive inductance losses.

The controller $\mathbf{K}^{1}$ has three inputs; the PCC voltage $v_{s}$, the grid current $i$ and the reference current $i^{*}$. The only measurements necessary for the controller implementation are, then, the grid currents and voltages, which means a reduced costs in sensors. Note, in addition, that the grid PCC voltage is usually available at no extra sensor cost as grid synchronisation algorithms use it. Dividing the controller matrix in rows $\mathbf{K}(s)=\left[\begin{array}{lll}K_{s}(s) & K_{r e f}(s) & K_{i}(s)\end{array}\right]^{T}$ results in the closedloop current dynamic shown below;

$$
\left.I\right|_{Z_{g}(s)=0}=\underbrace{\frac{G K_{r e f}}{1-G K_{i}}}_{T(s)} I^{*}+\underbrace{\frac{G_{d}+G K_{s}}{1-G K_{i}}}_{Y(s)} V_{s},
$$

\footnotetext{
${ }^{1}$ For notation compactness, the Laplace variable ' $s$ ' is omitted when its presence results obvious attending to the context
}

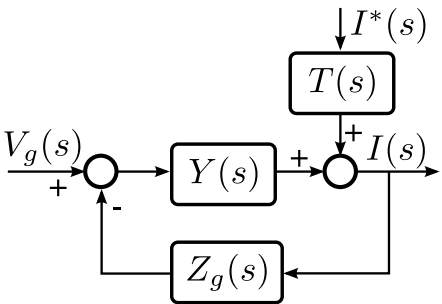

Fig. 2. Block diagram representation of the interconnection of a currentcontrolled VSC to a non-ideal grid with equivalent serial impedance $Z_{g}(s)$.

where $T(s)$ and $Y(s)$ are the closed-loop tracking and admittance transfer functions for an ideal grid (i.e., grid impedance $\left.Z_{g}(s)=0\right)$.

\section{B. Stability robustness of the Grid-VSC interaction}

When a current-controlled VSC is connected to an ideal grid, whose PCC voltage $v_{s}=v_{g}$ is independent of the grid current, system stability depends on the closed-loop system formed by the converter and the current controller, as defined (4). Assuming linearity, that amounts to place the roots of $1-G K_{i}$ in the left hand side of the complex plane, or to comply with one of the multiple equivalent formulations.

When this controlled-system is connected to a grid with non-negligible equivalent series grid impedance, the system behaves following the block diagram of Fig. 2, described by the following equations:

$$
\left.I\right|_{Z_{g}(s) \neq 0}=\underbrace{\frac{T}{1+Z_{g} Y}}_{T^{\prime}(s)} I^{*}+\underbrace{\frac{Y}{1+Z_{g} Y}}_{Y^{\prime}(s)} V_{g},
$$

where $V_{g}$ is the ideal grid voltage and $Y^{\prime}(s)$ and $T^{\prime}(s)$ are the modified closed-loop admittance and tracking transfer functions, respectively. From (5), it can be seen that the system stability no longer depends only on the stand-alone conditions, but also on the new impedances loop function $L^{\prime}(s)=Z_{g} Y$.

Provided that the system is stand-alone stable, the connection stability relies on the relationship between $Z_{g}$ and $Y$. Several criteria have been proposed in the literature [12], [28]. One particularly interesting approach is to take advantage of the Strictly Positive Realness of function $Z_{g}(s)$ [29]: if the rendered $Y$ is positive real, the product $Z_{g} Y$ will not get negative values, ensuring stability regardless of the functions respective modules.

A system that behaves like a low resistive admittance in a wide range will damp both grid voltage harmonic/interharmonics and the LCL filter resonance, in addition to the improved stability robustness in weak grids. Designing a current controller with good stand-alone stability margins that fulfils this condition is the main objective of this work.

\section{DESIGN PROCEDURE AND LIMITATIONS}

\section{A. Model-reference approach for controller design}

The desired controller is obtained following a model reference $\mathcal{H}_{\infty}$ design approach [23]. Readers can refer to previous 


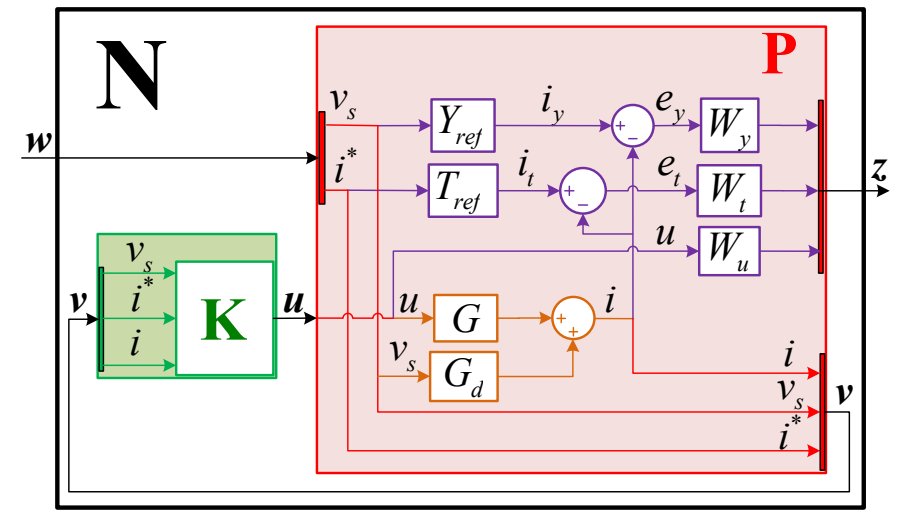

Fig. 3. $\mathcal{H}_{\infty}$ model-reference approach for admittance shaping control. In red the generalized plant $\mathbf{P}(s)$. Inside of it, the open loop command-to-output $G$ and admittance $G_{d}(s)$ transfer function, in orange. In purple, a set of elements added for the $\mathcal{H}_{\infty}$ controller synthesis. They serve as the design parameters. Finally, the obtained controller is represented in green.

work presented by the authors for a more detailed explanation of the underlying theory [24].

The keystone of the $\mathcal{H}_{\infty}$ control paradigm is the definition of the inner structure (i.e., its input and output vectors) of the generalized plant $\mathbf{P}$ [23]. Fig. 3 shows, in red, the structure of the plant for the presented method. It is formed by the open-loop current dynamic in (1), shown in orange, and the addition of some design parameters shown in purple. $\mathbf{P}$ has the next inputs and outputs; input vector $\boldsymbol{w}$, formed by the perturbation signals of the closed-loop system $v_{s}$ and $i^{*}$ (see equation (4)), input $u$ and output vector $\boldsymbol{v}$, which are equal to the inputs and outputs of the controller $\mathbf{K}$ output and inputs, respectively, and the output vector $z$, which is formed by the signals to be minimized in the design. Once the plant $\mathbf{P}$ is defined, an $\mathcal{H}_{\infty}$ synthesis process will be used to compute the controller that minimises the energy (norm-2) of the output vector $z$ with respect to the exogenous input vector $\boldsymbol{w}$ (i.e., the one that minimize $\left.\|\mathbf{N}\|_{\infty}\right)$.

In Fig. 3, $Y_{r e f}(j \omega)$ represents the desired behaviour, in the frequency domain, of the closed-loop admittance $Y(j \omega)$, as so does $T_{r e f}(j \omega)$ for the desired tracking transfer function $T(j \omega)$. Obtained controller should fulfil either $T(j \omega) \approx$ $T_{\text {ref }}(j \omega)$ or $Y(j \omega) \approx Y_{\text {ref }}(j \omega)$ at a given frequency, resulting in the minimization of the tracking shaping $e_{t}$ or admittance shaping $e_{y}$ error signals. Which one is fulfilled depends on the magnitude of the tracking $W_{t}(j \omega)$ and admittance $W_{y}(j \omega)$ frequency weights. If the magnitude of $W_{y}(j \omega)$ is higher than $W_{t}(j \omega)$ at a given frequency $\omega_{x}$ the synthesized controller $\mathbf{K}(j \omega)$ will fulfil $Y\left(j \omega_{x}\right) \approx Y_{r e f}\left(j \omega_{x}\right)$; tracking shaping works analogously. A third weight $W_{u}(j \omega)$ is added to limit the controller output (i.e., control effort) $u$. Similarly, high $W_{u}(j \omega)$ values will result in higher actuation limitation and vice-versa.

Fig. 4 summarizes the design process. First, the desired behaviour of the closed-loop tracking $T_{r e f}$ and admittance transfer functions $Y_{\text {ref }}$ are chosen. Next, controller objectives are divided in different frequency zones by means of the

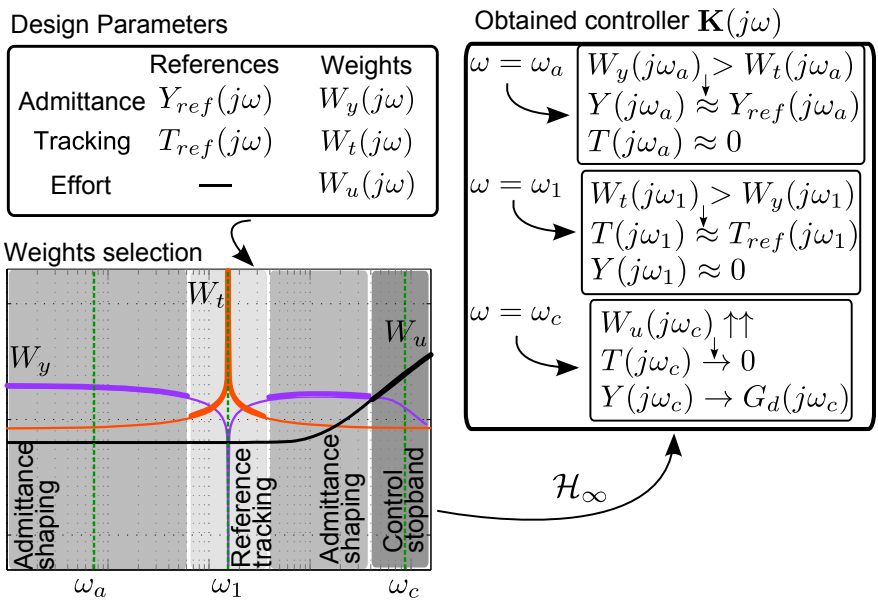

Fig. 4. Simplified scheme of the controller design process.

frequency shaped weights, as depicted in the weight selection of Fig. 4. In this work, controller $\mathbf{K}$ should result in a low resistive closed-loop admittance profile $Y(s)$, with good standalone stability margins and good tracking of the current reference at the grid fundamental frequency $\omega_{1}$ (i.e., $T\left(j \omega_{1}\right) \approx 1$ ).

\section{B. Design procedure}

$T_{\text {ref }}(s)$ is defined constant and equal to one, which will result in a good current tracking (i.e., $i \approx i^{*}$ ) at the desired frequencies. $Y_{r e f}(s)$ is defined constant (i.e., resistive) and with the lowest possible value. This value is usually, as will be justified later on, the open-loop admittance $G_{d}(j \omega)$ magnitude at the end of the control bandwidth.

For this design, a good admittance shaping (i.e., $Y(j \omega) \approx$ $\left.Y_{\text {ref }}(j \omega)\right)$ is desired at both sub- and super-synchronous frequencies and good tracking (i.e., $T(j \omega) \approx T_{\text {ref }}(j \omega)$ ) is desired around the grid synchronous (i.e., fundamental) frequency $\omega_{1}$. Additionally, the controller effort $u$, and then the controller bandwidth, must be limited at high frequencies. The next weighting functions are hence proposed below:

$$
\begin{aligned}
W_{t}(s) & =K_{t} \frac{s^{2}+2 \zeta_{n} \omega_{1} s+\omega_{1}^{2}}{s^{2}+2 \zeta_{d} \omega_{1} s+\omega_{1}^{2}} \\
W_{y}(s) & =K_{y} \frac{s^{2}+2 \zeta_{d} \omega_{1} s+\omega_{1}^{2}}{s^{2}+2 \zeta_{n} \omega_{1} s+\omega_{1}^{2}} \cdot \frac{1}{\left(1 / \omega_{y}\right) s+1} \\
W_{u}(s) & =K_{u} \frac{\left(1 / \omega_{u 1}\right) s+1}{\left(1 / \omega_{u 2}\right) s+1}
\end{aligned}
$$

A frequency domain magnitude representation of them is shown in Fig. 4. Tracking weight is formed by an initial low gain $K_{t}$ and a resonant-like gain centred at fundamental frequency $\omega_{1}$. Admittance weight is a low pass function with an initial gain $K_{y}>K_{t}$ and a pole at $\omega_{y}$ to limit the admittance shaping band. A notch is added at $\omega_{1}$, complementary to $W_{t}$ resonance, to improve the reference tracking shaping inside its frequency range. Finally, control effort weight $W_{u}$ is a highpass function with a small initial gain $K_{u}$, increasing at high frequencies by means of a zero in $\omega_{u 1}$. A pole at $\omega_{u 2}$ is added at high frequencies to make $W_{u}$ proper. 
Some heuristic rules that have been found to be useful for the weights parameters tuning are presented below. A graphical interpretation of them is summarised in Fig. 5:

1) Admittance shaping range: It is recommended to first tune the admittance weight $W_{y}$ parameters $K_{y}$ and $\omega_{y}$. These parameters will define the maximum frequency where tight admittance control is desired. It is important to respect the maximum bandwidth limits that will be described in the next subsection.

2) Actuation limitation range: The next step is to consider the controller effort weight $W_{u}$. The controller bandwidth is defined by the relative relation between $W_{y}$ and $W_{u}$. It is modified, then, by means of the zero at $\omega_{u 1}$, along with the previously fixed $W_{y}$ pole at $\omega_{y}$. The controller bandwidth must be kept inside certain limits to obtain good system stability margins. Additionally, the amount of controller effort $u$ within this bandwidth is controlled by the initial gain $K_{u}$ relative to $K_{y}$. This is adjusted to get a controller effort near the saturation limit under nominal transients and disturbances (e.g., voltage dips). That constitutes the first trade-off to be solved, between admittance error minimization and allowed controller actuation / stability robustness.

3) Tracking shaping range: The last step is the tuning of the reference tracking band. The tracking weight $W_{t}$ is included along with the complementary notch-like part in $W_{y}$. The minimization of the tracking shaping error $e_{t}$ at $\omega_{1}$ depends on the $W_{t}$ resonance height, which is the ratio $n_{h}=\zeta_{n} / \zeta_{d}$. The higher this ratio is, the smaller the error (i.e., perfect current tracking $i \approx i_{\text {ref }}$ can be obtained for very high $n_{h}$ values). The reference tracking bandwidth is mainly determine by the range where $W_{t}>W_{y}$. It can be modified by increasing the initial value $K_{t}$ in $W_{t}$ relative to the previously fixed initial value $K_{y}$ in $W_{y}$, or by increasing $\zeta_{n}$ value in both $W_{t}$ and $W_{y}$ to increase the resonance/notch width ${ }^{2}$. The wider this band is, the faster the current reference tracking will be, to the detriment of a reduced admittance shaping range around fundamental frequency. Moreover, as current reference tracking strongly relies on feedback action, an increase of the tracking bandwidth will also result in poorer stability margins. A compromise is hence needed in the tuning of these controller parameters.

\section{Method and controller limitations}

Elements in $\mathbf{P}$ must be LTI and proper. This implies that designed weights can not have infinite gain states (i.e., pure integrators/resonators); however, they can be placed arbitrarily close to the $j \omega$ axis.

The obtained controller $\mathbf{K}$ has the same order of $\mathbf{P}$, so an increase of the design parameters (i.e., weights or model references) order will provoke an equal increase of $\mathbf{K}$ order. Order reduction techniques can be used, if necessary, to reduce the computation burden of the implemented controller. Alg. 1 in [24] summarizes the modulator modelling process, generalized plant $\mathbf{P}$ generation, controller synthesis process and its discretization for its implementation in a digital controller.

\footnotetext{
${ }^{2}$ In order to preserve the ratio $n_{h}, \zeta_{d}$ must increase equally to $\zeta_{n}$
}

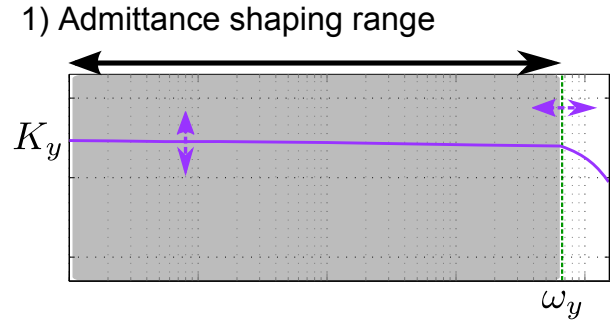

2) Actuation limitation range

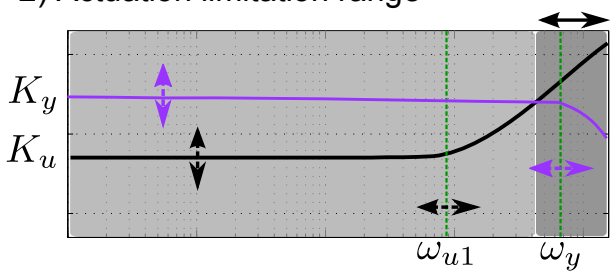

3) Tracking shaping range

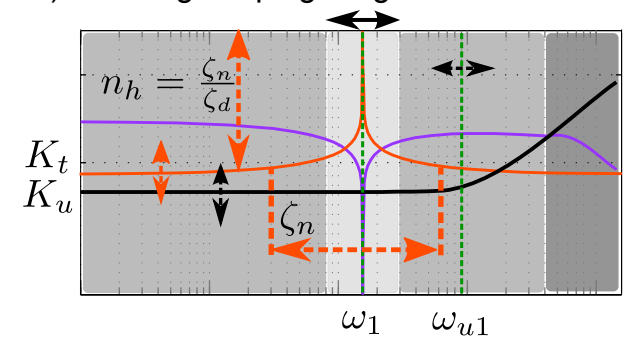

Fig. 5. Step-by-step weights design graphic guideline.

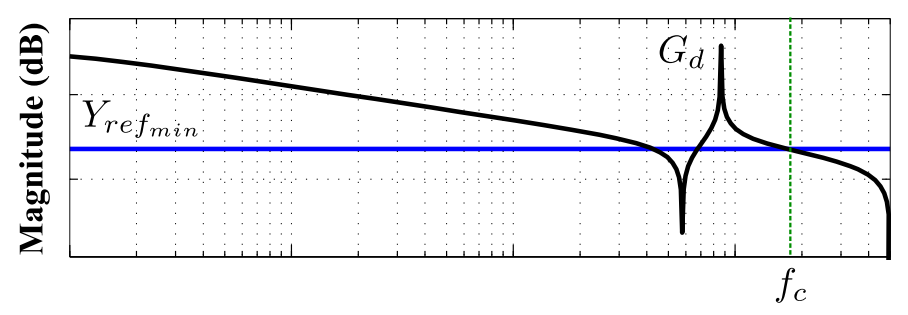

Fig. 6. Minimum obtainable resistive admittance reference $Y_{\text {ref }} f_{\text {min }}$ as a function of the open-loop admittance $G_{d}$ and the maximum admittance shaping bandwidth $f_{c}$.

The control of the system by PWM signals generated by a digital control platform induces bandwidth limitations. A high bound in controller bandwidth limitation is $f_{c}=1 /\left(2 \pi T_{s}\right)$ $\mathrm{Hz}$ [23]. It is important to note that this limitation affects both feedforward and feedback terms because both are affected by the modulator and the delay. This means that admittance shaping can not be obtained above this frequency, making impossible to reduce the closed-loop admittance $Y(s)$ below its open-loop value $G_{d}(s)$ at $f_{c}$. From an application design point of view, this implies that the minimum obtainable resistive admittance reference $Y_{\text {ref }}$ is equal to $G_{d}(s)$ magnitude at $f_{c}$, and, to reduce it, either the sampling time $T_{s}$ must be reduced or the filter inductances must be increased, as both will result in a smaller magnitude of $G_{d}(s)$ at $f_{c}$ (see Fig. 6).

The loop function $L(s)$ relative degree complies with the conditions for appliance of the Bode sensitivity integral theorem (first waterbed formula) [23]. It states that an increase in the feedback action of the controller inside a given frequency 
TABLE I. EXPERIMENTAL SETUP PARAMETERS

\begin{tabular}{lrlr}
\hline$V_{g N}$ & $120 \mathrm{~V}$ & $\omega_{1}$ & $2 \pi 60 \mathrm{rad} / \mathrm{s}$ \\
\hline$S_{n}$ & $2.6 \mathrm{kVA}$ & $C_{D C}$ & $600 \mu \mathrm{F}$ \\
\hline$V_{D C}^{*}$ & $650 \mathrm{~V}$ & $C$ & $15 \mu \mathrm{F}$ \\
\hline$L_{1}$ & $5.2 \mathrm{mH}$ & $L_{2}$ & $4 \mathrm{mH}$ \\
\hline$R_{1}$ & $28.8 \mathrm{~m} \Omega$ & $R_{2}$ & $18.6 \mathrm{~m} \Omega$ \\
\hline$T_{s}$ & $100 \mu \mathrm{s}$ & $T_{s w}$ & $100 \mu \mathrm{s}$ \\
\hline
\end{tabular}
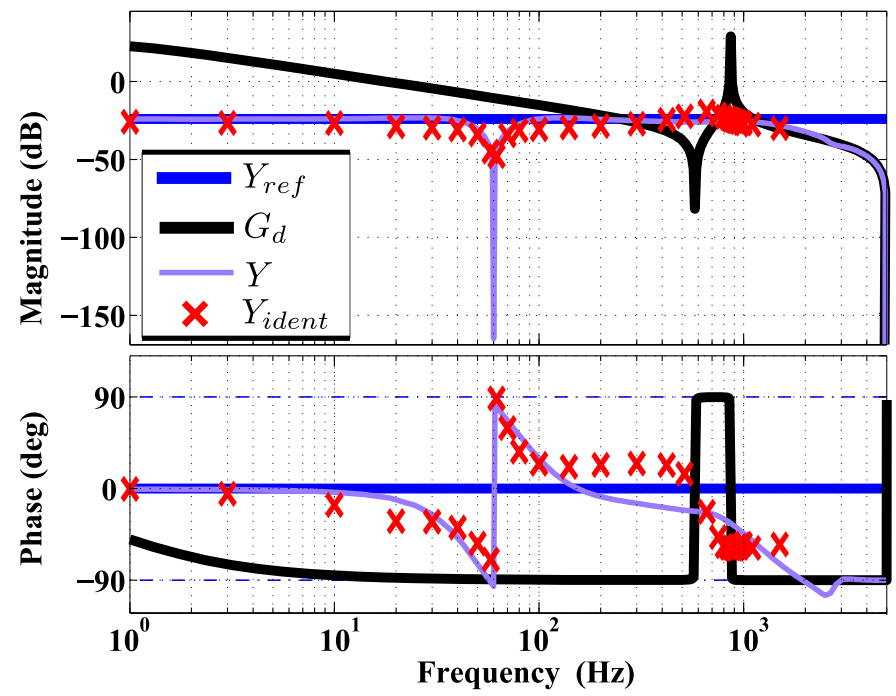

Fig. 7. Obtained input admittance by the proposed method.

band (i.e., frequencies where $|S(s)|<1$ ) comes at the cost of a higher $\|S(s)\|_{\infty}$ value and, then, in a reduction of the stability margins. Design parameters must take into account this tradeoff between close-loop performance and system robustness.

\section{RESUlts}

Table I shows the main parameters considered for the experimental set up. $S_{n}, V_{g N}$ and $\omega_{1}$ are the nominal power, grid voltage and grid frequency, respectively. $V_{D C}^{*}$ and $C_{D C}$ are the DC-bus reference voltage and capacitor value, respectively. Finally, $T_{s}$ is the sampling period of the digital controller and $T_{s w}$ is the switching period of the Danfoss VSC. The controller is implemented in a DS1007 dSPACE system. An AC programmable power supply Chroma 61845 is used to emulate the grid in order to generate different harmonics and inter-harmonics in the PCC.

\section{A. Frequency domain results}

Fig. 7 shows the obtained closed-loop admittance $Y$, its open-loop value $G_{d}$ and the given admittance reference $Y_{\text {ref }}$ in purple, black and blue lines respectively. Obtained admittance $Y$ follows given reference $Y_{r e f}=0.06 \Omega^{-1}$ in the desired frequencies (i.e., sub and super synchronous). $Y_{\text {ident }}$ shows experimental measurements of the controlled admittance ${ }^{3}$. The

\footnotetext{
${ }^{3}$ To obtain them, three phase small controlled voltage signals are added to the nominal grid voltage at different frequencies by means of the AC power supply. The steady-state current response is, then, measured. $Y_{\text {ident }}$ marks the magnitude/phase relation between the introduced voltage and measured current at each frequency.
}

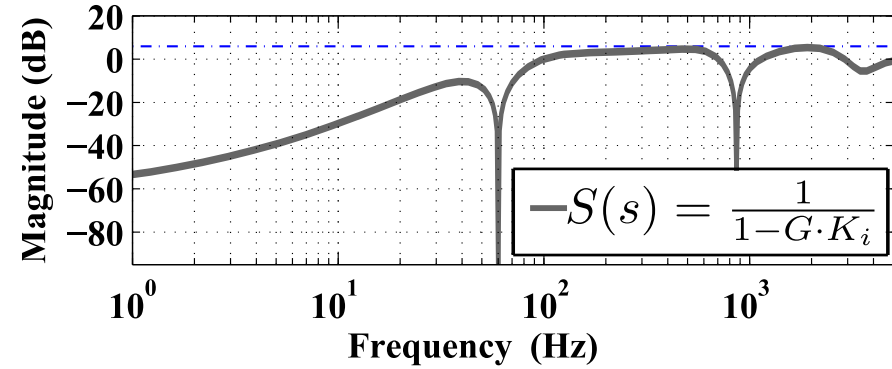

Fig. 8. Sensitivity function magnitude.

experimental admittance results show a well damped LCL resonance seen in $G_{d}$. Additionally, it demonstrates that any harmonic/inter-harmonics in the PCC will be damped to a current oscillation of, at most, $6 \%$ of the introduced voltage perturbation.

\section{B. Robustness results}

Fig. 8 shows the magnitude of the sensitivity transfer function $S(s)$. Its maximum peak value fulfils the stand-alone stability condition $\|S(s)\|_{\infty}<6 \mathrm{~dB}$, marked in the figure by a blue dashed line. As stated before, that will assure good stand-alone stability margins, with a gain margin $G M>6$ $\mathrm{dB}$ and a phase margin $P M>30^{\circ}$.

The presented application is next tested for two different grid impedance topologies: a purely inductive grid $Z_{g}(s)=$ $L_{g} s$ and a resonant inductive-capacitive grid $Z_{g}(s)=L_{g} s+$ $1 /\left(C_{g} s\right)$. Fig. 9 shows modified admittance $Y^{\prime}(s)$ closed loop poles (i.e., roots of $1+Z_{g} Y$ ) for changes of $L_{g}$ in the inductive grid and $C_{g}$ capacitance in the inductive-capacitive grid: $L_{g}$ is fixed to $0.11 \mathrm{pu}^{4}$ in the latter. The system is stable for all inductive grids considered ${ }^{5}$. However, it has some unstable zones for inductive-capacitive grids (red lines in Fig. 9). These instabilities appear when the resonance of $Z_{g}$ matches, in frequency, the two non-dissipative zones of the obtained admittance $Y$ in Fig. 7: the first in the fundamental frequency $\omega_{1}$, that interacts with the grid for a $C_{g}$ inside the interval $[9,10] \mathrm{pu}$, and the second at high frequencies outside the controller bandwidth $f_{c}$, for a $C_{g}$ inside the interval $[0.003,0.025] \mathrm{pu}$.

Fig. 10 and Fig. 11 show $Y^{\prime}(s)$ magnitude for some of the previous inductive and inductive-capacitive grid changes, respectively. As it can be seen, an inductive behaviour of the grid decreases even more the obtained admittance and don't affect the LCL resonance damping. However, the interaction between the $L C$ grid and the high frequency non-dissipative zone of the converter admittance generates a resonance in $Y^{\prime}$ : the red box in Fig. 11 represents the zone where this resonance is unstable. The capacitive behaviour of $Z_{g}$ interacts in this case with

\footnotetext{
${ }^{4}$ Grid impedance parameters are expressed in per unit values of the system nominal impedance $Z_{N}=V_{g N} / S_{N}$ (i.e., $L_{N}=Z_{N} / \omega_{1}$ and $\left.C_{N}=1 /\left(Z_{N} \omega_{1}\right)\right)$

${ }^{5}$ It is important to remark that some of these grid impedances are not feasible in practice, as their high values will require huge controller actuation for grid current tracking. This analysis only shows the stability robustness of the method.
} 

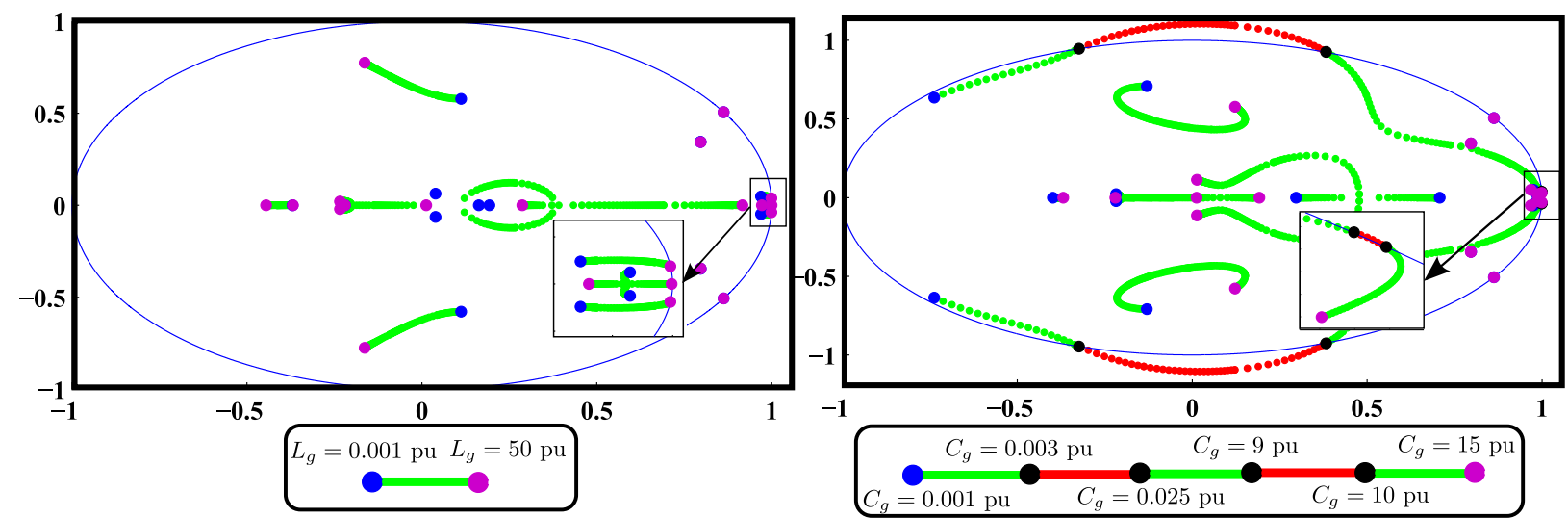

Fig. 9. The admittance $Y^{\prime}(s)$ poles for changes of inductive grid (left) and changes of capacitive grid (right). The red lines represents unstable cases.

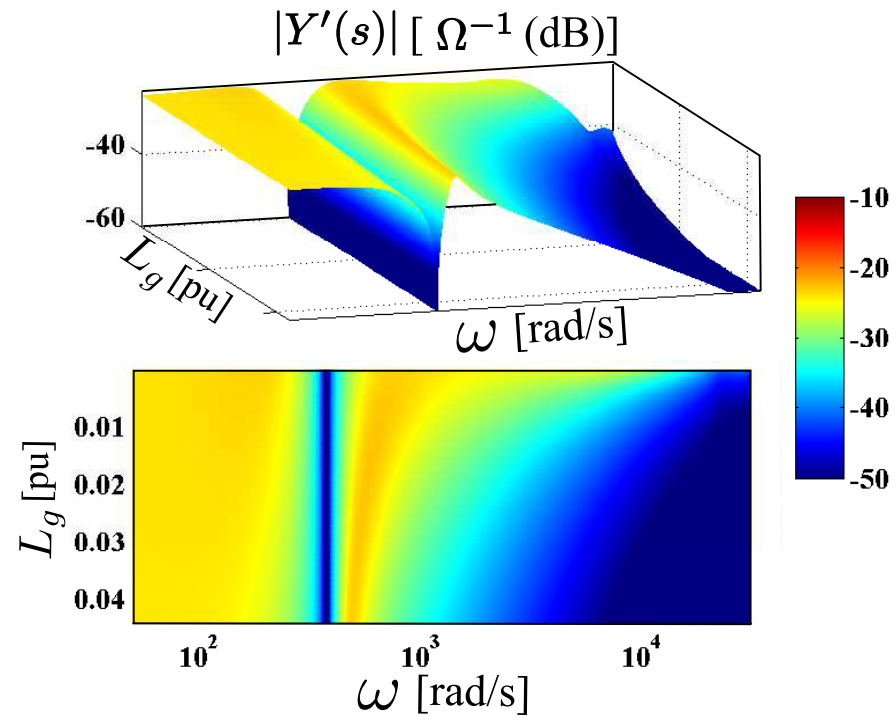

Fig. 10. Admittance magnitude $\left|Y^{\prime}(s)\right|$ for changes of an inductive grid.

the inductive and non-dissipative behaviour of the converter at high frequencies. As this non-dissipative zone is beyond the admittance control bandwidth, it can only be reduced by a more complex admittance reference that increases system admittance phase (and, then, magnitude) enough prior to the end of controller bandwidth. Some previous works have study this by including derivative terms in their current controllers [17], [30]. However, this phase increase will provoke bigger actuations and, if feedback is involved, smaller stand-alone margins. In any case, the result in Fig. 11 shows the worst case scenario, where the grid resonance is not damped by any resistive element, which is rarely the real case. Additionally, as shown in Fig. 7, experimental results $Y_{\exp }$ presents a more resistive behaviour at high frequencies than the theoretical obtained admittance $Y$, which means that this non-dissipative zone could even not exist in the real application.

\section{Time domain results}

This subsection presents some time domain experimental results of the obtained grid current control for different grid

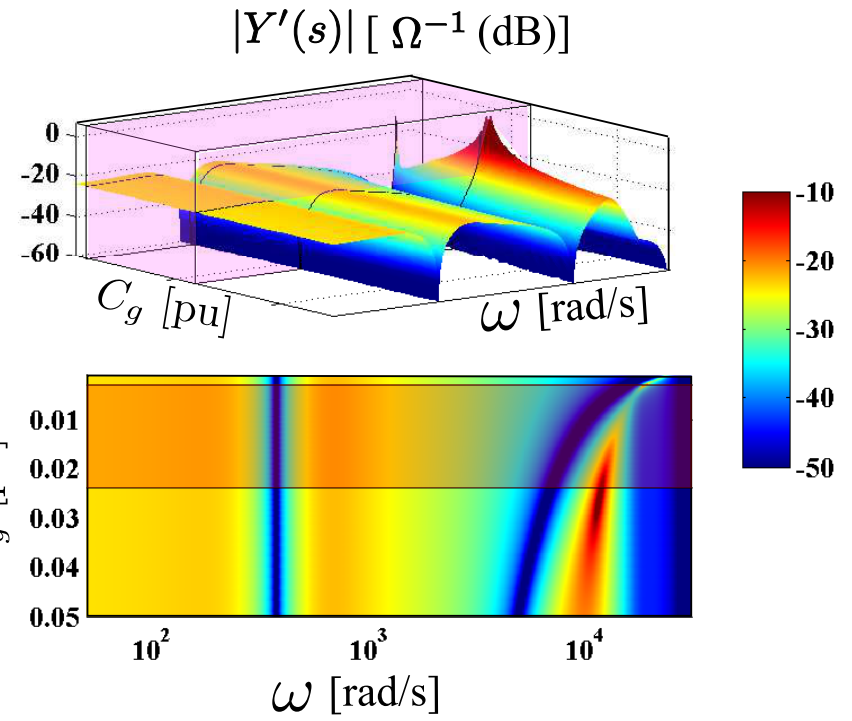

Fig. 11. Admittance magnitude $\left|Y^{\prime}(s)\right|$ for changes of an inductivecapacitive grid. The red box represents $C_{g}$ interval where the complex system becomes unstable.

conditions, grouped in Fig. 12. Fig. 12(a) shows the tracking results for a sudden change of the grid current reference $i^{*}$ with ideal grid conditions (i.e., $Z_{g}=0$ and PCC voltage $v_{s}$ equal to its nominal value). As it can be seen, the grid current successfully follows its reference. Fig. Fig. 12(b) shows a similar experiment, this time with an LC resonant grid impedance connected between the LCL filter and the AC power supply. Again, the current is stable and quickly track the given reference. Note that the grid impedance resonance is perfectly damped by the converter resistive admittance. This behaviour is expected attending to admittance magnitude $\left|Y^{\prime}(s)\right|$ in Fig. 11 for the chosen grid impedance parameters (i.e., $L_{g}=0.11 \mathrm{pu}$ and $C_{g}=0.05 \mathrm{pu}$ ).

The grid voltage harmonic rejection capabilities of the proposed method are tested below. Fig. 13 shows the total harmonic distortion (THD) for four different simulated test cases and different operational points: In test 1 (T1) a $5^{\text {th }}$ harmonic of $0.12 \mathrm{pu}$ is introduced in the grid; in test 2 (T2) 
(a)

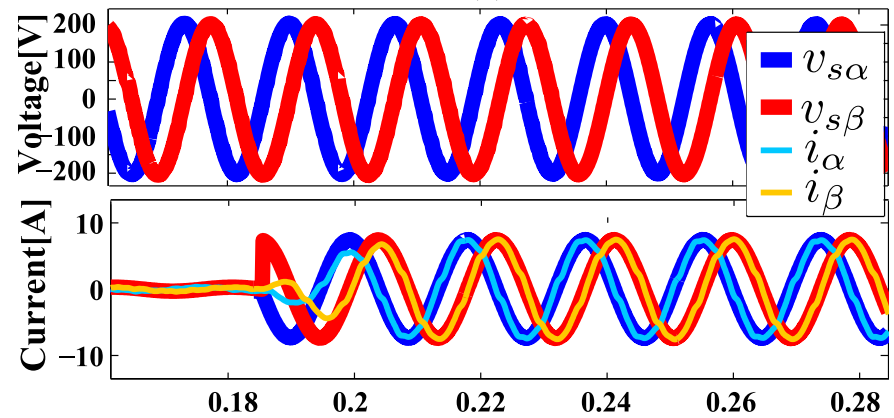

(b)

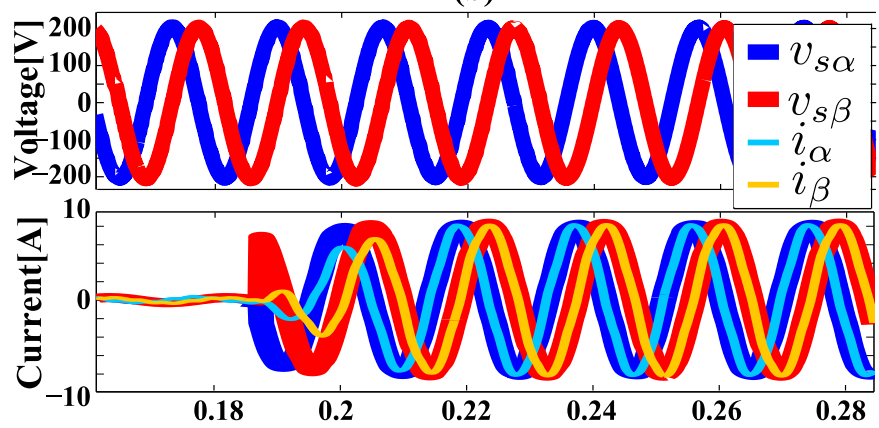

(c)

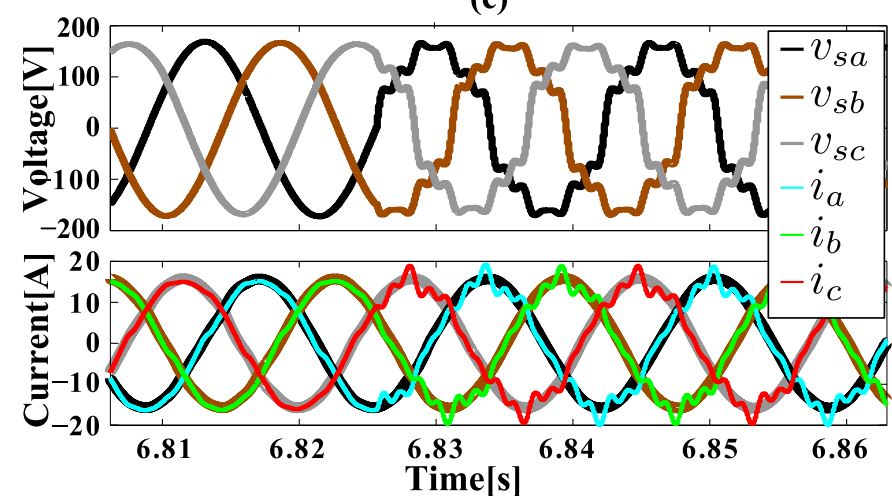

Fig. 12. (a) Experimental time domain tracking results for an ideal grid impedance $Z_{g}=0$. (b) Experimental time domain tracking results for a resonant LC grid: $L_{g}=0.11$ pu and $C_{g}=0.05$ pu. (c) Experimental time domain results to test 4 with an ideal grid impedance $Z_{g}=0$.

a $7^{\text {th }}$ harmonic of $0.1 \mathrm{pu}$ is added to T1; in test 3 (T3) two more harmonics, $11^{\text {th }}=0.7 \mathrm{pu}$ and $13^{\text {th }}=0.6 \mathrm{pu}$, are added; finally test 4 (T4) is completed with many high frequency harmonics $19^{\text {th }}=0.6 \mathrm{pu}, 23^{\text {th }}=0.6$ p.u, $25^{\text {th }}=0.6 \mathrm{pu}$, $29^{\text {th }}=0.5 \mathrm{pu}, 31^{\text {th }}=0.3 \mathrm{pu}, 35^{\text {th }}=0.3 \mathrm{pu}$ and $37^{\text {th }}=0.3$ pu. Fig. 12(c) shows the experimental time domain results to test 4 . The grid currents $i$ still follows its (reactive) reference $i^{*}$ after the introduction of grid voltage harmonics in $v_{s}$, with reduced current distortion thanks to its low admittance profile.

\section{CONCLUSION}

A grid current controller for a VSC connected to the grid through an LCL filter using a shaped closed loop admittance is presented. With the presented method, a low resistive admittance can be obtained, which will simultaneously damp the filter resonance and reduce the effect of grid voltage

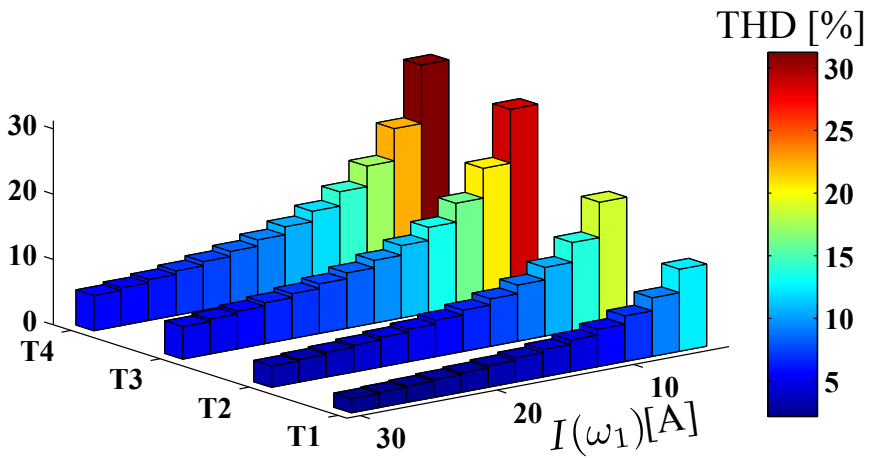

Fig. 13. Obtained THD for different harmonic test.

harmonics and inter-harmonics in a wide frequency range, even at high frequencies closed to the filter resonance. In terms of damping, the main advantage of the method is the reduced number of sensors needed to its implementation. In terms of harmonics damping, the presented method is probably not as effective as the classical PR gains tuned at certain frequencies (if a good system modelling is considered, including discretization and time delays effects); the main advantage of the method is that it damps the disturbance from the grid voltage in a wider frequency range, both harmonics and inter-harmonics and in both sub and super synchronous frequencies. Additionally, the system has good stand-alone stability margins, which is a known concern when multiple PR feedback gains are introduced. Finally, the low resistive admittance obtained makes the system very robust for changes of the grid impedance.

Future studies will analyse the effect of more complex admittance profiles, whit bigger magnitude reduction at the main grid harmonic frequencies, to improve the current controller response to the main PCC voltage perturbations.

\section{ACKNOWLEDGEMENT}

The work of the University of Alcala group was supported in part by the spanish research projects CONPOSITE (ENE2011-28527-C04-02 Ministerio de Economía y Competitividad), PRICAM (S2013/ICE-2933 Consejería de educación, juventud y deporte de la Comunidad de Madrid) and DIANA(CCG2015/EXP-064 Universidad de Alcalá). The work of Robert Griñó was supported in part by the Government of Spain through the Ministerio de Economía y Competitividad Project DPI2013-41224-P and by the Generalitat de Catalunya through the project 2014 SGR 267.

\section{REFERENCES}

[1] L. Harnefors, L. Zhang, and M. Bongiorno, "Frequency-domain passivity-based current controller design," IET Power Electronics, vol. 1, no. 4, pp. 455-465, 2008.

[2] M. Liserre, R. Teodorescu, and F. Blaabjerg, "Stability of photovoltaic and wind turbine grid-connected inverters for a large set of grid impedance values," IEEE transactions on power electronics, vol. 21, no. 1, pp. 263-272, 2006.

[3] M. Liserre, A. D. Aquila, and F. Blaabjerg, "Genetic algorithm-based design of the active damping for an lcl-filter three-phase active rectifier," IEEE Transactions on Power Electronics, vol. 19, no. 1, pp. 76-86, 2004. 
[4] V. Blasko and V. Kaura, "A novel control to actively damp resonance in input lc filter of a three-phase voltage source converter," IEEE Transactions on Industry Applications, vol. 33, no. 2, pp. 542-550, 1997.

[5] J. L. Agorreta, M. Borrega, J. López, and L. Marroyo, "Modeling and control of-paralleled grid-connected inverters with lcl filter coupled due to grid impedance in pv plants," IEEE Transactions on Power Electronics, vol. 26, no. 3, pp. 770-785, 2011.

[6] J. Dannehl, F. W. Fuchs, S. Hansen, and P. B. Thøgersen, "Investigation of active damping approaches for pi-based current control of gridconnected pulse width modulation converters with lcl filters," IEEE Transactions on Industry Applications, vol. 46, no. 4, pp. 1509-1517, 2010.

[7] J. He and Y. W. Li, "Generalized closed-loop control schemes with embedded virtual impedances for voltage source converters with lc or lcl filters," IEEE Transactions on Power Electronics, vol. 27, no. 4, pp. 1850-1861, 2012.

[8] Y. Tang, P. C. Loh, P. Wang, F. H. Choo, and F. Gao, "Exploring inherent damping characteristic of lcl-filters for three-phase grid-connected voltage source inverters," IEEE Transactions on Power Electronics, vol. 27, no. 3, pp. 1433-1443, 2012.

[9] S. G. Parker, B. P. McGrath, and D. G. Holmes, "Regions of active damping control for lcl filters," IEEE Transactions on Industry Applications, vol. 50, no. 1, pp. 424-432, 2014.

[10] T. Nussbaumer, M. L. Heldwein, and J. W. Kolar, "Differential mode input filter design for a three-phase buck-type pwm rectifier based on modeling of the emc test receiver,' IEEE Transactions on Industrial Electronics, vol. 53, no. 5, pp. 1649-1661, 2006.

[11] M. Cespedes, L. Xing, and J. Sun, "Constant-power load system stabilization by passive damping," IEEE Transactions on Power Electronics, vol. 26, no. 7, pp. 1832-1836, 2011.

[12] R. D. Middlebrook, "Input filter considerations in design and application of switching regulators," in Proc. of IAS, 1976.

[13] R. Pena-Alzola, M. Liserre, F. Blaabjerg, R. Sebastián, J. Dannehl, and F. W. Fuchs, "Analysis of the passive damping losses in lcl-filter-based grid converters," IEEE Transactions on Power Electronics, vol. 28, no. 6 , pp. 2642-2646, 2013

[14] R. W. Erickson, "Optimal single resistor damping of input filters," in Proc. of Applied Power Electronics Conference and Exposition, vol. 2. Citeseer, 1999, pp. 1073-1079.

[15] F. Rodriguez, E. Bueno, M. Aredes, L. Rolim, F. A. Neves, and M. C. Cavalcanti, "Discrete-time implementation of second order generalized integrators for grid converters," in Proc. of 34th Annual Conference on IEEE Industrial Electronics Society IECON 2008. IEEE, 2008, pp. $176-181$.

[16] A. Vidal, F. D. Freijedo, A. Yepes, P. Fernandez-Comesana, J. Malvar, O. Lopez, and J. Doval-Gandoy, "Assessment and optimization of the transient response of proportional-resonant current controllers for distributed power generation systems," IEEE Transactions on Industrial Electronics, vol. 60, no. 4, pp. 1367-1383, 2013.
[17] L. Harnefors, A. Yepes, A. Vidal, and J. Doval-Gandoy, "Passivity-based controller design of grid-connected vscs for prevention of electrical resonance instability,' IEEE Transactions on Industrial Electronics, vol. 62, no. 2, pp. 702-710, 2015

[18] A. G. Yepes, "Digital resonant current controllers for voltage source converters," Ph.D. dissertation, UNIVERSITY OF VIGO, 2011

[19] A. G. Yepes, F. D. Freijedo, O. Lopez, and J. Doval-Gandoy, "Analysis and design of resonant current controllers for voltage-source converters by means of nyquist diagrams and sensitivity function," IEEE Transactions on Industrial Electronics, vol. 58, no. 11, pp. 5231-5250, 2011.

[20] X. Wang, F. Blaabjerg, and P. C. Loh, "Virtual rc damping of 1clfiltered voltage source converters with extended selective harmonic compensation," IEEE Transactions on Power Electronics, vol. 30, no. 9, pp. 4726-4737, 2015.

[21] S. Cobreces, E. J. Bueno, F. J. Rodriguez, D. Pizarro, and F. Huerta, "Robust loop-shaping h-inf control of lcl-connected grid converters," in Proc. of IEEE International Symposium on Industrial Electronics (ISIE). IEEE, 2010, pp. 3011-3017.

[22] I. J. Gabe, V. F. Montagner, and H. Pinheiro, "Design and implementation of a robust current controller for vsi connected to the grid through an lcl filter," IEEE Transactions on Power Electronics, vol. 24, no. 6 , pp. 1444-1452, 2009.

[23] S. Skogestad and I. Postlethwaite, Multivariable feedback control: analysis and design. Wiley New York, 2007, vol. 2.

[24] J. Perez, S. Cobreces, R. Grino, and F. Rodriguez, "H-inf current controller for input admittance shaping of vsc-based grid applications," IEEE Transactions on Power Electronics, vol. PP, 2016.

[25] J. Sun, "Impedance-based stability criterion for grid-connected inverters," IEEE Transactions on Power Electronics, vol. 26, no. 11, pp. 30753078, 2011

[26] J. Perez, S. Cobreces, D. Pizarro, F. J. Rodriguez Sanchez, and R. Grino, "Resonance damping of lcl filters via input admittance frequency shaping," in Proc. of the 2016 IEEE 25th International Symposium on Industrial Electronics (ISIE). IEEE, 2016, pp. 516-521.

[27] P. C. Krause, O. Wasynczuk, and S. D. Sudhoff, Analysis of Electric Machinery and Drive Systems. 2nd Edition. IEEE Press. WileyInterscience, 2002.

[28] L. Harnefors, M. Bongiorno, and S. Lundberg, "Input-admittance calculation and shaping for controlled voltage-source converters," IEEE Transactions on Industrial Electronics, vol. 54, no. 6, pp. 3323-3334, 2007.

[29] S. V. Brian D. O. Anderson, Network Analysis and Synthesis: A Modern Systems Theory Approach. Dover Publications, 2006.

[30] X. Wang, F. Blaabjerg, and P. C. Loh, "Proportional derivative based stabilizing control of paralleled grid converters with cables in renwable power plants," in Proc. of IEEE Energy Conversion Congress and Exposition (ECCE). IEEE, 2014, pp. 4917-4924. 